\title{
The association between cancer-related fatigue and diabetes from pre-chemotherapy to 6-months post- chemotherapy
}

\section{Amber Kleckner ( $\square$ amber_kleckner@urmc.rochester.edu )}

University of Rochester Medical Center https://orcid.org/0000-0002-5088-1139

lan R. Kleckner

University of Rochester Medical Center

\section{Eva Culakova}

University of Rochester Medical Center

\section{Michelle Shayne}

University of Rochester Medical Center

\section{Elizabeth K. Belcher}

University of Rochester Medical Center

\section{Abdi Gudina}

University of Rochester Medical Center

\section{AnnaLynn M. Williams}

St Jude Children's Research Hospital

\section{Adedayo A. Onitilo}

Marshfield Medical Center

Judith 0 . Hopkins

Novant Health

\section{Howard Gross}

Dayton Physicians Network

\section{Karen M. Mustian}

University of Rochester Medical Center

\section{Luke J. Peppone}

University of Rochester Medical Center

Michelle C. Janelsins

University of Rochester Medical Center

\section{Research Article}

Keywords: Cancer-related fatigue, Chemotherapy, Diabetes, Metabolism, Supportive care

Posted Date: February 12th, 2021

DOI: https://doi.org/10.21203/rs.3.rs-220601/v1 
License: (우 This work is licensed under a Creative Commons Attribution 4.0 International License. Read Full License 


\section{Abstract}

\section{Purpose}

To quantify the impact of diabetes on the trajectory of cancer-related fatigue (CRF) from pre-chemotherapy to 6 months post-chemotherapy for patients with breast cancer compared to non-cancer controls.

\section{Methods}

This was a secondary analysis from a nationwide prospective longitudinal study of female patients with breast cancer undergoing chemotherapy and age-matched women without cancer (controls). CRF was measured using the Multidimensional Fatigue Symptom Inventory (MFSI) pre-, post-, and 6-months post-chemotherapy in patients; controls were assessed at equivalent time points. Diabetes status was obtained at baseline. Repeated measures mixed models estimated the association between CRF and diabetes controlling for cancer $(y / n)$, body mass index, exercise and smoking habits, baseline anxiety and depressive symptoms, menopausal status, marital status, race, and education.

\section{Results}

A total of 439 patients and 235 controls (age: $52.8 \pm 10.5$ years) had available data on diabetes status. Diabetes was twice as prevalent among patients as controls ( $11.6 \%$ vs. $6.8 \%)$. Patients had worse fatigue than controls throughout treatment $(p<0.001)$. Diabetes was associated with worse CRF with a clinically meaningful difference of $4.7 \pm 1.7$ points on the fatigue measure in all participants $(p=0.009)$ and patients alone $(p=0.030)$. For MFSI subdomains, diabetes was associated with worse general $(p=0.002)$, physical $(p=0.005)$, and mental fatigue $(p=0.025)$ but not worse emotional fatigue or vigor $(p>0.14)$ among patients.

\section{Conclusions}

Diabetes was twice as prevalent in women with breast cancer compared to controls, and diabetes was associated with more severe CRF in patients before and after chemotherapy and at 6 months postchemotherapy. Interventions that address diabetes management may also help address CRF during chemotherapy treatment

\section{Introduction}

Cancer-related fatigue affects at least $30-90 \%$ of patients [1-3]. Fatigue can also persist long after the cessation of treatment, as approximately one-third of cancer survivors experience fatigue up to 6 years after cancer treatment $[4,5]$. By definition, cancer-related fatigue is chronic, not proportional to recent activity, and not relieved by additional sleep or rest [6]. Its severity can impair the ability to perform activities of daily living, greatly reduce quality of life, and increase mortality [6-8]. There are currently few effective preventive strategies or treatments for cancer-related fatigue, in part because its etiology and pathophysiology are still being characterized. Cancerrelated fatigue has multifactorial etiology including psychological and biological factors, and mechanisms include, increasingly, metabolic dysfunction [9-11]. For example, obesity, a prevalent metabolic co-morbidity, is a known risk factor for cancer-related fatigue $[6,12,13]$. 
Diabetes mellitus is a common metabolic disorder and one of the most common chronic conditions in the United States with a prevalence of at least $10.5 \%$ of all Americans [14]. At the same time, the coexistence of diabetes and cancer, specifically breast cancer, is increasingly common. Diabetes and poor glycemic control are risk factors for morbidity from cancer and its treatments. For example, in a study by Srokowski et al., patients with stage I-III breast cancer and diabetes were 1.4-times more likely to be hospitalized for chemotherapy toxicities than patients without diabetes [15]. In a study among patients with non-Hodgkin's lymphoma, patients with diabetes needed more chemotherapy dose adjustments, had more delays between cycles, and had fewer cycles because of severe side effects compared to patients without diabetes [16]. While glycemic control does not always worsen with cancer treatment [17], ancillary medications that are commonly prescribed with chemotherapy such as steroids can interfere with glycemic control and even induce diabetes mellitus [18]. There is also substantial co-occurrence between symptoms of diabetes, symptoms of cancer, and side effects of treatment [19]. As such, diabetes is sometimes associated with increased prevalence and severity of specific symptoms/side effects including fatigue, pain, cognitive impairment, anxiety, depression, and chemotherapyinduced peripheral neuropathy, some of which are pre-existing and some of which develop during cancer treatment (review [20]). Specifically, in a cross-sectional study of 6,188 female breast cancer survivors in China, diabetes was associated with significantly more severe fatigue, but there was no comparison to people who did not have cancer. The shared symptomatic burden and potentially overlapping metabolic mechanisms of cancerrelated fatigue and diabetes support a rationale that diabetes might exacerbate cancer-related fatigue before, during, and after treatment with chemotherapy.

We hypothesized that diabetes would be associated with more severe cancer-related fatigue during curative chemotherapy treatment for breast cancer and into early survivorship. Using data from a large, nationwide longitudinal trial that followed female patients with breast cancer from pre-chemotherapy to six months postchemotherapy, we assessed whether diabetes was associated with worse fatigue controlling for relevant demographics, clinical characteristics, and lifestyle behaviors. We also assessed whether diabetes status was associated with a greater change in fatigue from baseline to post-chemotherapy and baseline to 6 months after chemotherapy treatment.

\section{Methods}

\section{Study design and participants}

An observational cohort study was conducted through the University of Rochester Cancer Center (URCC) National Cancer Institute (NCI) Community Oncology Research Program (NCORP) Research Base to assess the trajectory of chemotherapy-induced toxicities among breast cancer patients at pre-chemotherapy, postchemotherapy, and six months post-chemotherapy; age- and gender-matched controls without cancer provided data at equivalent time points (URCC 10055; trial registration number: NCT01382082). The full methods and primary aims of the parent study have been published previously $[21,22]$. In the parent study, patients were recruited from 22 NCORP locations across the United States from 2011-2013.

This was a secondary analysis to assess the effects of diabetes on the trajectory of cancer-related fatigue at pre-, post-, and 6 months post-chemotherapy. In this study, participants had to be female, have a diagnosis of stage I to IIIC breast cancer, be scheduled for chemotherapy, be chemotherapy naïve, be at least 21 years of age, have no central nervous system or neurodegenerative diseases, have no recent major psychiatric illness leading 
to hospitalization, and have no plan to receive radiation therapy concurrent with their chemotherapy. Additionally, for this analysis, data had to be available for diabetes and fatigue status. Control participants were matched within five years of age to the patient and met all eligibility criteria except for the cancer diagnosis. Assessment time points occurred at baseline (within the seven days prior to the first chemotherapy administration), post-chemotherapy (within one month of the last chemotherapy administration), and six months post-chemotherapy (within a one-month range), or at equivalent time points for control participants. Participants were excluded from this analysis if diabetes status and/or MFSI data were not available at baseline (of 943 total participants, 269 total participants were excluded; 141 patients with cancer and 128 controls did not have diabetes status and 24 participants did not have MFSI data at baseline).

\section{Assessment of fatigue}

Patient-reported fatigue was assessed using the Multidimensional Fatigue Symptom Inventory-Short Form (MFSI-SF), which has demonstrated validity among patients with cancer [23]. The MFSI-SF is a 30-item questionnaire that inquires about how true each statement has been over the last seven days and requests a response on a five-point scale from 0 , "Not at all," to 4, "Very much." The questionnaire yields a final total score that ranges from -24 to 96 with a greater score indicated greater fatigue. The MFSI-SF also allows for the measurement of five specific subdomains of cancer-related fatigue-general fatigue, physical fatigue, mental fatigue, emotional fatigue, and vigor; for the first four subdomains, a higher score indicates worse fatigue, whereas a higher vigor score indicates less fatigue.

\section{Assessment of demographics and clinical characteristics including diabetes}

Demographic information, clinical characteristics, and lifestyle behaviors were obtained from medical records and/or self-report via study-specific forms at baseline. Diabetes diagnosis was captured as a binary variable (no, not diagnosed with diabetes, or yes, diagnosed with diabetes). Body mass index (BMI) was calculated from height and weight at baseline and was used as a continuous variable. Exercise was determined from selfreported current exercise habits (no or yes = planned physical activity performed to increase physical fitness 3-5 times per week for 20-60 minutes per session at a level that increases breathing rate and induces sweating). Smoking was also considered as a categorical variable and was determined using self-reported habits, where Never $=$ smoked $<100$ cigarettes in their lifetime, Former $=$ smoked $\geq 100$ cigarettes in their lifetime but do not smoke currently, and Current = currently in the habit of smoking cigarettes. Current menopausal status was coded categorically as pre-menopausal, peri-menopausal, post-menopausal, or medically induced. Marital status was determined using self-reported living situation (long term relationship [LTR] = married and living with spouse or long-term committed relationship and noLTR = single, widowed, separated, or divorced). Race was selfreported and was analyzed as a binary variable (White or Non-White). Education was considered a binary variable (<High school, High school graduate, or GED; or At least some college). Hypertension was assessed as a diagnosis of hypertension and was categorized as yes or no. Anxiety was assessed using the Spielberger Trait Anxiety Inventory [24]; depressive symptoms were assessed using a single item, "I feel depressed," with responses "not at all," "a little bit," somewhat," "quite a bit," or "very much;" and reading ability, a proxy for 
cognitive reserve, was assessed using the Wide Range Assessment Test-Fourth Edition (WRAT-4) reading subscale; all were treated as continuous variables [21]. There was $<5 \%$ missing data for all covariates.

\section{Statistics}

All analyses were performed using SAS (version 9.4, SAS Institute, Cary, NC) and JMP Pro (v. 14.1.0, SAS Institute). The distribution of baseline characteristics was evaluated for those with and without diabetes, and the mean (standard deviation) and n (\%) were reported for continuous and categorical measures, respectively.

Baseline correlations were conducted to determine the effects of cancer and diabetes on fatigue before chemotherapy using linear regression. To determine the effect of diabetes on the cancer-related fatigue as measured using total MFSI score over all three time points, a mixed model was developed using a repeated compound symmetry structure. Relevant covariates were identified from the literature and defined a priori-group (individuals with cancer receiving chemotherapy or individuals without cancer [control]), assessment time point (pre-chemotherapy, post-chemotherapy, 6 months post-chemotherapy), age, BMI, exercise, and smoking were fixed in the model. Other potential covariates-menopausal status, marital status, race, education, hypertension, anxiety, depression, and cognitive reserve-were added sequentially to assess their effects on the parameter estimate for the influence of diabetes on cancer-related fatigue $\left(\beta_{\text {Diab }}\right)$. If the $p$-value for the covariate was $<0.1$ and/or the effect estimate for diabetes changed more than $10 \%$, the covariate was retained in the model. Groupxtime was also fixed in the model. To evaluate the association between diabetes and cancer-related fatigue within group (cancer or control), a groupxdiabetes interaction term was added to each model, and then least square mean estimates were assessed for all pairwise comparisons (Student's $t$ ) for both cancer (diabetes yes or no) and control (diabetes yes or no). To assess the effects of diabetes on fatigue at post-chemotherapy and 6 months post-chemotherapy among patients only, an analysis of covariance (ANCOVA) was performed with baseline fatigue score as a covariate in addition to the other potential covariates as described above. The primary outcome measure of this secondary analysis was MFSI, total score. Because we also evaluated the effect of diabetes on the subdomains of the MFSI, we acknowledge that we performed multiple comparisons and there is risk of type 1 error, but this secondary analysis is hypothesis-generating by nature and results should be applied prudently and followed up upon in future hypothesis-testing studies. For this analysis, a p-value of $<0.05$ was deemed statistically significant.

\section{Results}

Among 674 total participants, 439 were patients with cancer and 235 were controls. See Table 1 for a description of the demographics, clinical characteristics, and lifestyle habits of the cohort. Participants were $52.8 \pm 10.5$ years old, $69.6 \%$ were overweight or obese, $73.3 \%$ were married or in a long-term relationship, $90.5 \%$ were White, and $81.9 \%$ had at least some college education. Among patients, $26.2 \%$ had stage I cancer, $49.9 \%$ had stage II, and $18.7 \%$ had stage III. A total of 51 patients with cancer had had a diagnosis of diabetes (11.6\%), and 16 controls had diabetes $\left(6.8 \%, p=0.007, \chi^{2}\right.$ test $)$.

\section{Baseline associations between diabetes and fatigue}


Figure 1 illustrates the unadjusted total MFSI fatigue scores for patients and controls with and without diabetes. At baseline (i.e., before chemotherapy treatment), patients had a greater level of fatigue than controls ( $\beta \pm S E=$ $4.00 \pm 0.97, p<0.001$ ), and all participants (patients and controls) with diabetes had a greater level of fatigue than those without diabetes $(\beta \pm S E=3.56 \pm 1.48, p=0.017$; Supplemental Tables 1A-D). Anxiety explained $35.1 \%$ of the variance in fatigue, followed by depressive symptoms $\left(\eta^{2}{ }_{p}=17.4 \%\right)$. Cancer status explained $2.53 \%$ of variance, age explained $1.25 \%$, and diabetes explained $0.88 \%$, which was more than menopausal status, exercise habits, $\mathrm{BMI}$, and smoking status $(0.68 \%, 0.62 \%, 0.38 \%$, and $0.32 \%$, respectively). When an interaction term was added to the model (Group×Diabetes), the term was not statistically significant $(p=0.54)$, suggesting having a cancer diagnosis and diabetes are each independent risk factors for fatigue.

\section{The effect of diabetes on total fatigue and fatigue subdomains from baseline to post-chemotherapy and six months post- chemotherapy}

When incorporating data from all three time points, diabetes was significantly associated with worse fatigue after adjusting for cancer status, time, age, BMI, exercise habits, smoking habits, marital status, menopausal status, baseline anxiety, and baseline depression as well as several other relevant covariates (Table 2, $\beta \pm S E=$ $4.72 \pm 1.7, p=0.006)$, and this relationship held true among cancer patients only $(\beta \pm S E=4.33 \pm 1.98, p=0.030)$ but not controls only due to the larger variation in fatigue values among controls $(\beta \pm S E=5.81 \pm 3.27, p=0.077)$. Diabetes was associated with worse fatigue on all five subdomains of the MFSI and reached statistical significance for general fatigue $(p=0.002)$, physical fatigue $(p=0.005)$, and mental fatigue $(p=0.025)$, though differences did not reach statistical significance for emotional fatigue $(p=0.639)$ or vigor $(p=0.105)$. Statistical significance was retained in patients only for general $(p=0.004)$ and mental fatigue $(p=0.022)$. Supplementary Tables 2A-F depict the final models relating diabetes and fatigue with the appropriate covariates for each of the five MFSI subdomains. An interaction between cancer status and diabetes was not seen for the total MFSI score $(p=0.696)$ or for any of the MFSI subdomains ( $p>0.209)$, suggesting that diabetes and cancer are independent contributors to fatigue (Supplemental Tables 3A- F).

\section{The effect of diabetes on changes in fatigue over the course of chemotherapy treatment}

Next, we performed multivariate regression models to assess the differences in the change in fatigue (MFSI total) from baseline to post-chemotherapy and baseline to 6 months post-chemotherapy among those with and without diabetes. We did not observe significant differences in the worsening of fatigue (i.e., change over time) from baseline to post-chemotherapy between those with and without diabetes in all participants $(p=0.45)$ or among patients only $(p=0.84)$. Similarly, we did not observe significant differences in the change in fatigue from baseline to 6 months post-chemotherapy between those with and without diabetes in all participants $(p=0.22)$ or in patients only $(p=0.12)$, suggesting that those with diabetes recovered to a similar level as those without diabetes by 6 months post-chemotherapy treatment (Supplemental Tables 4A-F). There was also no interaction effect between cancer and diabetes $(p=0.67$ from baseline to post-chemotherapy and $p=0.58$ from baseline to 6 
months post-chemotherapy), corroborating our previous findings that diabetes and cancer contribute to fatigue independently.

\section{Discussion}

This is one of the first studies to investigate the interrelationship between diabetes and cancer-related fatigue using a longitudinal study with both patients undergoing chemotherapy for cancer treatment and individuals without cancer. We showed that diabetes was significantly more prevalent among patients than individuals without cancer, diabetes is associated with more severe fatigue to a clinically meaningful degree, and diabetes and the cancer experience are independent contributors to fatigue, especially general, physical, and mental fatigue.

These data corroborate our prior findings that cancer is associated with significant fatigue especially after chemotherapy [25] and, additionally, that fatigue is a common symptom among patients with diabetes without cancer [19]. Our data also build upon findings of Hammer et al. from 244 patients with solid tumors undergoing chemotherapy [26], in which patients were categorized into those without diabetes, with pre-diabetes, and with diabetes according to baseline glycosylated hemoglobin concentrations $\left(\mathrm{Hb}_{\mathrm{A} 1 \mathrm{c}}\right)$; those with pre-diabetes and diabetes had clinically meaningful greater fatigue in the morning than those without diabetes, and those with diabetes had greater fatigue in the evening, though differences were not statistically significant [26]. Our data, in a larger sample size ( $n=439$ patients with cancer plus 235 controls) and different study design, confirmed this relationship and achieved statistical significance using a different measure of fatigue (MFSI herein vs. Lee Fatigue Scale).

These data suggest that clinical control of diabetes is important during cancer treatment. In one study ([28]), though not all studies (e.g., [29]), adherence to diabetes medications declined from $75 \%$ to $25 \%$ during treatment for breast cancer, resulting in elevated $\mathrm{Hb}_{\mathrm{A} 1 \mathrm{c}}$. Low adherence was associated with more hospitalizations [29]. Unfortunately, we did not have access to medication adherence, clinical control of diabetes, or $\mathrm{Hb}_{\mathrm{A} 1 \mathrm{c}}$, but it is possible that poor diabetes medication adherence could be associated with worse fatigue. In addition, antiemetics and steroids are commonly prescribed in conjunction with chemotherapy and can adversely affect glycemic control. Metformin, a common drug to stabilize blood glucose among people with diabetes, is a promising antineoplastic agent $[30,31]$ and future research should assess its efficacy to ameliorate supportive care outcomes.

Our results corroborate previous literature that describes risk factors for fatigue (review [6]) and interventional studies treating fatigue-namely age (age was associated with less fatigue), exercise habits (regular exercise protected against fatigue) [6], baseline anxiety and depression (strong positive correlations) [6, 32], menopausal status (medically induced menopause was associated with the worst fatigue, followed by peri- and postmenopausal status, followed by pre-menopausal status) [33, 34], BMI (obesity was associated with worse fatigue than normal weight, especially for physical fatigue, e.g., [6, 12]), and smoking habits (current smoking was associated with worse fatigue) [35]. With the exception of age and menopausal status, these risk factors can all be addressed via behavioral interventions and highlight the potential for lifestyle interventions (e.g., nutrition, exercise, smoking cessation) to ameliorate fatigue before and during treatment via direct or indirect effects on metabolic health. 
Despite lack of a multiplicative interaction between cancer and diabetes, it is still possible that metabolic dysfunction, including glucose regulation, plays a role in the etiology and pathophysiology of cancer-related fatigue. In a study of patients with breast cancer undergoing chemotherapy, patients experienced a worsening of all aspects of metabolic syndrome including fasting blood glucose, insulin, and $\mathrm{Hb}_{\mathrm{A} 1 \mathrm{c}}$ [36] (fatigue was not reported in this trial). Metabolic dysfunction can stem from cancer- or treatment-related inflammation or neuroendocrine dysfunction, resulting in dysregulation of insulin and other hormones [9]. Metabolic dysfunction can also result from direct effects of chemotherapy on mitochondrial bioenergetics that can reduce ATP energy production, especially in muscle (reviews $[37,38]$ ). Exploration into the chemotherapy-induced pathology of mitochondria in the presence and absence of diabetes [39] and overall metabolic function of pancreatic $\beta$-cells should be the topic of future research.

This study is strong in that it is one of the first to specifically explore the relationships between diabetes and cancer-related fatigue by comparing results in patients with cancer to fatigue and diabetes in those who do not have cancer. It involves a large sample of patients with breast cancer from community oncology clinics throughout the United States, which allows for generalizability of the results. It also compares patients to individuals who do not have cancer, allowing us to discern the degree of fatigue that is associated specifically with the cancer experience. Our primary outcome, the MFSI, is the gold standard for fatigue measurement [40] and is validated among patients with cancer [23]. Our ability to assess fatigue across three important time points during the cancer experience permitted longitudinal analyses through which we can account for the variability in fatigue over time among controls as well as assess changes in fatigue from before chemotherapy to just after and six months after chemotherapy.

However, this study has certain limitations. For example, we could not distinguish between Type 1 and Type 2 diabetes mellitus, which have different pathophysiology, nor did we identify people with pre-diabetes, which could have been a substantial proportion of our population; similarly, we did not assess $\mathrm{Hb}_{\mathrm{A} 1 \mathrm{c}}$ or $\mathrm{HOMA}-\mathrm{IR}$ to validate diabetes status. Additionally, for most controls, medical record verification of diabetes status was not available.

Despite this, we still observed an association and the true risk of diabetes on fatigue is likely larger than what we observed. In addition, exercise and smoking habits were self-report, and participants might be more inclined to report healthier habits than actual. This would lead to non-differential misclassification and therefore more conservative effect parameters than actual. Further, BMI served to control for excess fat mass, though it is not always an accurate surrogate [41]. Lastly, this study was predominately White, highly educated, non-smoking women, so our data should not be generalized to other populations without prudence. This was a secondary analysis, so our observed relationships between diabetes and cancer-related fatigue should be tested for replication in future research.

In conclusion, diabetes was twice as prevalent among patients with cancer as non-cancer controls. Also, diabetes was associated with cancer-related fatigue to a clinically meaningful degree before, during, and after chemotherapy treatment for female breast cancer patients after controlling for demographics, clinical characteristics, and lifestyle factors. If these results are true, glycemic control during the cancer experience could reduce the burden of acute and long-term cancer-related fatigue. Future research is needed to elucidate the mechanisms underlying fatigue so that we can develop metabolically targeted therapies. In the meantime, it is important for clinicians to encourage metabolic health, perhaps via healthy lifestyle practices (e.g., diet, exercise, 
healthy sleep habits, not smoking) as soon as possible in the cancer continuum in order to reduce the burden of diabetes on fatigue.

\section{Declarations}

Funding: This research was supported by NIH NCI UG1CA189961 to KMM and Gary Morrow, NCI T32CA102618 to MCJ and Gary Morrow, NCI R01CA231014 to MCJ, and NCI K07CA221931 to IRK.

Conflicts of interest/Competing interests: Authors report no conflicts of interest.

Ethics approval: Ethical approval was obtained from Institutional Review Boards at URCC and each of the 22 recruitment sites prior to enrollment. This trial was performed in accordance with the ethical standards of the 1964 Helsinki declaration and its later amendments or comparable ethical standards.

Consent to participate: Informed consent was obtained from all individual participants included in the study.

Consent for publication: N/A

Availability of data and code: The datasets and statistical code generated during and analyzed during the current study are available from the corresponding author on reasonable request.

\section{References}

[1] Berger AM, Mooney K, Alvarez-Perez A, et al. (2015) Cancer-Related Fatigue, Version 2.2015, Clinical Practice Guidelines in Oncology. Nat Comp Cancer Netw 13: 1012-1039

[2] Servaes P, Verhagen C, Bleijenberg G (2002) Fatigue in cancer patients during and after treatment: prevalence, correlates and interventions. European Journal of Cancer 38: 27-43

[3] Al Maqbali M, Al Sinani M, Al Naamani Z, Al Badi K, Tanash MI (2020) Prevalence of Fatigue in Patients with Cancer: A Systematic Review and Meta-Analysis. J Pain Symptom Manage

[4] Jones JM, Olson K, Catton P, et al. (2016) Cancer-related fatigue and associated disability in post-treatment cancer survivors. J Cancer Surviv 10: 51-61

[5] Bower JE, Wiley J, Petersen L, Irwin MR, Cole SW, Ganz PA (2018) Fatigue after breast cancer treatment: Biobehavioral predictors of fatigue trajectories. Health Psychol 37: 1025-1034

[6] Bower JE (2014) Cancer-related fatigue-mechanisms, risk factors, and treatments. Nature Reviews Clinical Oncology 11: 597-609

[7] Horneber M, Fischer I, Dimeo F, Ruffer JU, Weis J (2012) Cancer-related fatigue: epidemiology, pathogenesis, diagnosis, and treatment. Dtsch Arztebl Int 109: 161-171; quiz 172

[8] Hofman M, Ryan JL, Figueroa-Moseley CD, Jean-Pierre P, Morrow GR (2007) Cancer-related fatigue: the scale of the problem. Oncologist 12 Suppl 1: 4-10 
[9] Saligan LN, Olson K, Filler K, et al. (2015) The biology of cancer-related fatigue: a review of the literature. Support Care Cancer 23: 2461-2478

[10] O'Higgins CM, Brady B, O'Connor B, Walsh D, Reilly RB (2018) The pathophysiology of cancer-related fatigue: current controversies. Support Care Cancer 26: 3353-3364

[11] Grossberg AJ, Vichaya EG, Gross PS, et al. (2020) Interleukin 6-independent metabolic reprogramming as a driver of cancer-related fatigue. Brain Behav Immun 88: 230-241

[12] Inglis JE, Janelsins MC, Culakova E, et al. (2020) Longitudinal assessment of the impact of higher body mass index on cancer-related fatigue in patients with breast cancer receiving chemotherapy. Support Care Cancer

[13] Aleixo GFP, Deal AM, Nyrop KA, et al. (2020) Association of body composition with function in women with early breast cancer. Breast Cancer Res Treat 181: 411-421

[14] American Diabetes Association (2017) CDC National Diabetes Statistics Report, 2017. In, Arlington, VA

[15] Srokowski TP, Fang S, Hortobagyi GN, Giordano SH (2009) Impact of diabetes mellitus on complications and outcomes of adjuvant chemotherapy in older patients with breast cancer. J Clin Oncol 27: 2170-2176

[16] van Herpt TTW, van de Schans SAM, Haak HR, van Spronsen DJ, Dercksen MW, Janssen-Heijnen MLG (2011) Treatment and outcome in non-Hodgkin's lymphoma patients with and without prevalent diabetes mellitus in a population-based cancer registry. Journal of Geriatric Oncology 2: 239-245

[17] Karlin NJ, Dueck AC, Nagl Reddy SK, Verona PM, Cook CB (2014) Implications of breast cancer with diabetes mellitus on patient outcomes and care. Diabetes Management 4: 1-9

[18] Lee SY, Kurita N, Yokoyama Y, et al. (2014) Glucocorticoid-induced diabetes mellitus in patients with lymphoma treated with CHOP chemotherapy. Support Care Cancer 22: 1385-1390

[19] Singh R, Teel C, Sabus C, McGinnis P, Kluding P (2016) Fatigue in Type 2 Diabetes: Impact on Quality of Life and Predictors. PLoS One 11: e0165652

[20] Storey S, Cohee A, Gathirua-Mwangi WG, et al. (2019) Impact of Diabetes on the Symptoms of Breast Cancer Survivors. Oncol Nurs Forum 46: 473-484

[21] Janelsins MC, Heckler CE, Peppone LJ, et al. (2018) Longitudinal Trajectory and Characterization of CancerRelated Cognitive Impairment in a Nationwide Cohort Study. Journal of Clinical Oncology 32: 3231-3239

[22] Janelsins MC, Heckler CE, Peppone LJ, et al. (2017) Cognitive Complaints in Survivors of Breast Cancer after Chemotherapy Compared with Age-Matched Controls: An Analysis From a Nationwide, Multicenter, Prospective Longitudinal Study. J Clin Oncol 35: 506-514

[23] Stein KD, Jacobsen PB, Blanchard CM, Thors C (2004) Further validation of the multidimensional fatigue symptom inventory-short form. Journal of Pain and Symptom Management 27: 14-23 
[24] Spielberger CD, Sydeman SJ, Owen AE, et al. (1999) Measuring anxiety and anger with the State-Trait Anxiety Inventory (STAI) and the State-Trait Anger Expression Inventory (STAXI). In: Maruish ME (ed) The Use of Psychological Testing for Treatment Planning and Outcomes Assessment. Lawrence Erlbaum Associates, Mahwah, NJ, pp 993-1021

[25] Williams AM, Paterson C, Heckler CE, et al. (in press) Cancer-related fatigue, anxiety, and quality of life in breast cancer patients compared to non-cancer controls: A nationwide longitudinal analysis. Breast Cancer Reserach and Treatment

[26] Hammer MJ, Aouizerat BE, Schmidt BL, Cartwright F, Wright F, Miaskowski C (2015) Glycosylated Hemoglobin A1c and Lack of Association With Symptom Severity in Patients Undergoing Chemotherapy for Solid Tumors. Oncol Nurs Forum 42: 581-590

[27] Chan A, Yo TE, Wang XJ, et al. (2018) Minimal Clinically Important Difference of the Multidimensional Fatigue Symptom Inventory-Short Form (MFSI-SF) for Fatigue Worsening in Asian Breast Cancer Patients. J Pain Symptom Manage 55: 992-997 e992

[28] Calip GS, Hubbard RA, Stergachis A, Malone KE, Gralow JR, Boudreau DM (2015) Adherence to oral diabetes medications and glycemic control during and following breast cancer treatment. Pharmacoepidemiol Drug Saf 24: 75-85

[29] Tan X, Feng X, Chang J, Higa G, Wang L, Leslie D (2016) Oral antidiabetic drug use and associated health outcomes in cancer patients. J Clin Pharm Ther 41: 524-531

[30] Samuel SM, Varghese E, Varghese S, Busselberg D (2018) Challenges and perspectives in the treatment of diabetes associated breast cancer. Cancer Treat Rev 70: 98-111

[31] Evans JMM, Donnelly LA, Emslie-Smoth AM, Alessi DR, Morris AD (2005) Metformin and reduced risk of cancer in diabetic patients. BMJ 330: 1304-1305

[32] Oh HS, Seo WS (2011) Systematic review and meta-analysis of the correlates of cancer-related fatigue. Worldviews Evid Based Nurs 8: 191-201

[33] Tchen N, Juffs HG, Downie FP, et al. (2003) Cognitive function, fatigue, and menopausal symptoms in women receiving adjuvant chemotherapy for breast cancer. J Clin Oncol 21: 4175-4183

[34] Mar Fan HG, Houede-Tchen N, Chemerynsky I, et al. (2010) Menopausal symptoms in women undergoing chemotherapy-induced and natural menopause: a prospective controlled study. Ann Oncol 21: 983-987

[35] Peppone LJ, Mustian KM, Morrow GR, et al. (2011) The effect of cigarette smoking on cancer treatmentrelated side effects. Oncologist 16: 1784-1792

[36] Dieli-Conwright CM, Wong L, Waliany S, Bernstein L, Salehian B, Mortimer JE (2016) An observational study to examine changes in metabolic syndrome components in patients with breast cancer receiving neoadjuvant or adjuvant chemotherapy. Cancer 122: 2646-2653 
[37] Yang S, Chu S, Gao Y, et al. (2019) A Narrative Review of Cancer-Related Fatigue (CRF) and Its Possible Pathogenesis. Cells 8

[38] Sorensen JC, Cheregi BD, Timpani CA, Nurgali K, Hayes A, Rybalka E (2016) Mitochondria: Inadvertent targets in chemotherapy-induced skeletal muscle toxicity and wasting? Cancer chemotherapy and pharmacology 78: 673-683

[39] Las G, Oliveira MF, Shirihai OS (2020) Emerging roles of beta-cell mitochondria in type-2-diabetes. Mol Aspects Med 71: 100843

[40] Schvartsman G, Park M, Liu DD, Yennu S, Bruera E, Hui D (2017) Could Objective Tests Be Used to Measure Fatigue in Patients With Advanced Cancer? J Pain Symptom Manage 54: 237-244

[41] Prentice AM, Jebb SA (2001) Beyond body mass index. Obesity Reviews 2: 141-147

\section{Tables}

Table 1. Demographics and clinical characteristics 


\begin{tabular}{|c|c|c|c|c|c|}
\hline Characteristic & $\begin{array}{l}\text { Total } \\
\text { participants } \\
(n=674)\end{array}$ & $\begin{array}{l}\text { Cancer with } \\
\text { diabetes } \\
(n=51)\end{array}$ & $\begin{array}{l}\text { Cancer without } \\
\text { diabetes } \\
(n=388)\end{array}$ & $\begin{array}{l}\text { Control with } \\
\text { diabetes } \\
(n=16)\end{array}$ & $\begin{array}{l}\text { Control without } \\
\text { diabetes } \\
(n=219)\end{array}$ \\
\hline Age & $52.8 \pm 10.5$ & $58.6 \pm 8.6$ & $52.5 \pm 10.7$ & $53.3 \pm 9.8$ & $52.0 \pm 10.2$ \\
\hline \multicolumn{6}{|l|}{ Body mass index } \\
\hline $\begin{array}{l}\text { Underweight or } \\
\text { normal weight }(<25 \\
\left.\mathrm{kg} / \mathrm{m}^{2}\right)\end{array}$ & $202(30.0 \%)$ & $5(9.8 \%)$ & $122(31.4 \%)$ & $3(18.8 \%)$ & $72(33.3 \%)$ \\
\hline $\begin{array}{l}\text { Overweight }(\geq 25 \\
\left.\text { and }<30 \mathrm{~kg} / \mathrm{m}^{2}\right)\end{array}$ & $180(26.7 \%)$ & $6(11.8 \%)$ & $95(24.5 \%)$ & $2(12.5 \%)$ & 77 (35.6\%) \\
\hline Obese $\left(\geq 30 \mathrm{~kg} / \mathrm{m}^{2}\right)$ & $289(42.9 \%)$ & $40(78.4 \%)$ & $171(44.1 \%)$ & $11(68.8 \%)$ & $67(31.0 \%)$ \\
\hline \multicolumn{6}{|l|}{ Regular exercise } \\
\hline Yes & $334(49.6 \%)$ & $20(39.2 \%)$ & $180(46.4 \%)$ & $5(31.3 \%)$ & $129(59.7 \%)$ \\
\hline No & $340(50.4 \%)$ & $31(60.8 \%)$ & $208(53.6 \%)$ & $11(68.8 \%)$ & $90(41.7 \%)$ \\
\hline \multicolumn{6}{|l|}{ Smoking habits } \\
\hline Never & $403(59.8 \%)$ & $34(66.7 \%)$ & $219(56.4 \%)$ & $9(56.3 \%)$ & $141(65.3 \%)$ \\
\hline Former & $198(29.4 \%)$ & $16(31.4 \%)$ & $117(30.2 \%)$ & $6(37.5 \%)$ & $59(27.3 \%)$ \\
\hline Current & $64(9.5 \%)$ & $1(2.0 \%)$ & $47(12.1 \%)$ & $1(6.3 \%)$ & $15(6.9 \%)$ \\
\hline \multicolumn{6}{|l|}{ Menopausal status } \\
\hline Pre-menopausal & $209(31.0 \%)$ & $4(7.8 \%)$ & $136(35.1 \%)$ & $4(25.0 \%)$ & $65(30.1 \%)$ \\
\hline Post-menopausal & $346(51.3 \%)$ & $34(66.7 \%)$ & $200(51.5 \%)$ & $7(43.8 \%)$ & $105(48.6 \%)$ \\
\hline Peri-menopausal & $67(9.9 \%)$ & $6(11.8 \%)$ & $27(7.0 \%)$ & $3(18.8 \%)$ & $31(14.4 \%)$ \\
\hline Medically induced & $52(7.7 \%)$ & $7(13.7 \%)$ & $25(6.4 \%)$ & $2(12.5 \%)$ & $18(8.3 \%)$ \\
\hline \multicolumn{6}{|l|}{ Marital status } \\
\hline $\begin{array}{l}\text { Married/long-term } \\
\text { relationship }\end{array}$ & 494 (73.3\%) & $33(64.7 \%)$ & $289(74.5 \%)$ & $8(50.0 \%)$ & $164(75.9 \%)$ \\
\hline $\begin{array}{l}\text { Single, divorced, } \\
\text { widowed, separated }\end{array}$ & $180(26.7 \%)$ & $18(35.3 \%)$ & 99 (25.5\%) & $8(50.0 \%)$ & $55(25.5 \%)$ \\
\hline \multicolumn{6}{|l|}{ Race } \\
\hline White & $610(90.5 \%)$ & 37 (72.5\%) & $354(91.2 \%)$ & $15(93.8 \%)$ & $204(94.4 \%)$ \\
\hline Other & 64 (9.5\%) & $14(27.5 \%)$ & $34(8.8 \%)$ & $1(6.3 \%)$ & 15 (6.9\%) \\
\hline \multicolumn{6}{|l|}{ Education } \\
\hline $\begin{array}{l}\text { High school/GED or } \\
\text { less }\end{array}$ & $122(18.1 \%)$ & 15 (29.4\%) & $84(21.6 \%)$ & $3(18.8 \%)$ & $20(9.3 \%)$ \\
\hline At least some & 552 (81.9\%) & $36(70.6 \%)$ & 304 (78.4\%) & $13(81.3 \%)$ & 199 (92.1\%) \\
\hline
\end{tabular}


college

Hypertension

\begin{tabular}{llllll} 
Yes & $194(28.8 \%)$ & $35(68.6 \%)$ & $99(25.5 \%)$ & $9(56.3 \%)$ & $51(23.6 \%)$ \\
\hline No & $479(71.1 \%)$ & $16(31.4 \%)$ & $288(74.2 \%)$ & $7(43.8 \%)$ & $168(77.8 \%)$ \\
\hline Baseline anxiety* & $33.4 \pm 12.0$ & $36.9 \pm 13.3$ & $36.0 \pm 12.3$ & $26.8 \pm 5.7$ & $28.4 \pm 9.8$ \\
$\begin{array}{l}\text { Baseline } \\
\text { depressiont }\end{array}$ & $0.58 \pm 0.87$ & $0.73 \pm 0.92$ & $0.67 \pm 0.91$ & $0.75 \pm 1.13$ & $0.37 \pm 0.72$ \\
$\begin{array}{l}\text { Baseline cognitive } \\
\text { reservef }\end{array}$ & $63.1 \pm 5.4$ & $61.5 \pm 7.7$ & $62.8 \pm 5.7$ & $64.4 \pm 4.1$ & $63.8 \pm 4.1$ \\
\hline
\end{tabular}

Cancer stage

\begin{tabular}{lll} 
I & $15(29.4 \%)$ & $100(25.8 \%)$ \\
II & $21(41.2 \%)$ & $198(51.0 \%)$ \\
III Unknown & $11(21.6 \%)$ & $71(18.3 \%)$ \\
\hline
\end{tabular}

*Anxiety was measured using the Spielberger Trait Anxiety Inventory tDepression was assessed using item 21 on the Multidimensional Fatigue Symptom Inventory-Short Form ¥Reading ability, a proxy for cognitive reserve, was assessed using the Wide Range Assessment Test-Fourth Edition (WRAT-4) reading subscale

Table 2. The association between diabetes and cancer-related fatigue*

\begin{tabular}{|c|c|c|c|c|c|c|c|c|c|}
\hline & \multicolumn{3}{|c|}{ All participants $(n=674)$} & \multicolumn{3}{|c|}{ Patients only ( $n=439$ ) } & \multicolumn{3}{|c|}{ Controls only $(n=235)$} \\
\hline & $\begin{array}{l}\text { Mean } \\
\text { difference }^{\dagger}\end{array}$ & SE & $\begin{array}{l}\mathrm{p}- \\
\text { value }\end{array}$ & $\begin{array}{l}\text { Mean } \\
\text { difference }\end{array}$ & SE & $\begin{array}{l}\mathrm{p}- \\
\text { value }\end{array}$ & $\begin{array}{l}\text { Mean } \\
\text { difference }\end{array}$ & SE & $\begin{array}{l}\mathrm{p}- \\
\text { value }\end{array}$ \\
\hline $\begin{array}{l}\text { Total } \\
\text { MFSI }\end{array}$ & 4.716 & 1.711 & $0.006^{\ddagger}$ & 4.325 & 1.983 & $0.030^{\ddagger}$ & 5.805 & 3.273 & 0.077 \\
\hline $\begin{array}{l}\text { General } \\
\text { fatigue }\end{array}$ & 1.812 & 0.594 & $0.002^{\ddagger}$ & 1.988 & 0.691 & $0.004^{\ddagger}$ & 1.749 & 1.127 & 0.121 \\
\hline $\begin{array}{l}\text { Physical } \\
\text { fatigue }\end{array}$ & 1.280 & 0.457 & $0.005^{\ddagger}$ & 0.945 & 0.529 & 0.075 & 2.222 & 0.878 & $0.012^{\ddagger}$ \\
\hline $\begin{array}{l}\text { Mental } \\
\text { fatigue }\end{array}$ & 1.034 & 0.460 & $0.025^{\ddagger}$ & 1.223 & 0.533 & $0.022^{\ddagger}$ & 0.500 & 0.883 & 0.572 \\
\hline $\begin{array}{l}\text { Emotional } \\
\text { fatigue }\end{array}$ & 0.153 & 0.326 & 0.639 & 0.171 & 0.376 & 0.648 & 0.018 & 0.612 & 0.976 \\
\hline Vigor & -0.739 & 0.455 & 0.105 & -0.529 & 0.529 & 0.318 & -1.284 & 0.867 & 0.139 \\
\hline
\end{tabular}

*Models were adjusted as described in the Methods section for age, race, marital status, education, body mass index, menopausal status, exercise habits, smoking habits, anxiety, depression, and/or cognitive reserve 
†Mean difference estimates denote the difference between participants with diabetes $(n=67)$ and participants without diabetes $(n=607)$

$\ddagger \mathrm{p}<0.05$

Figures

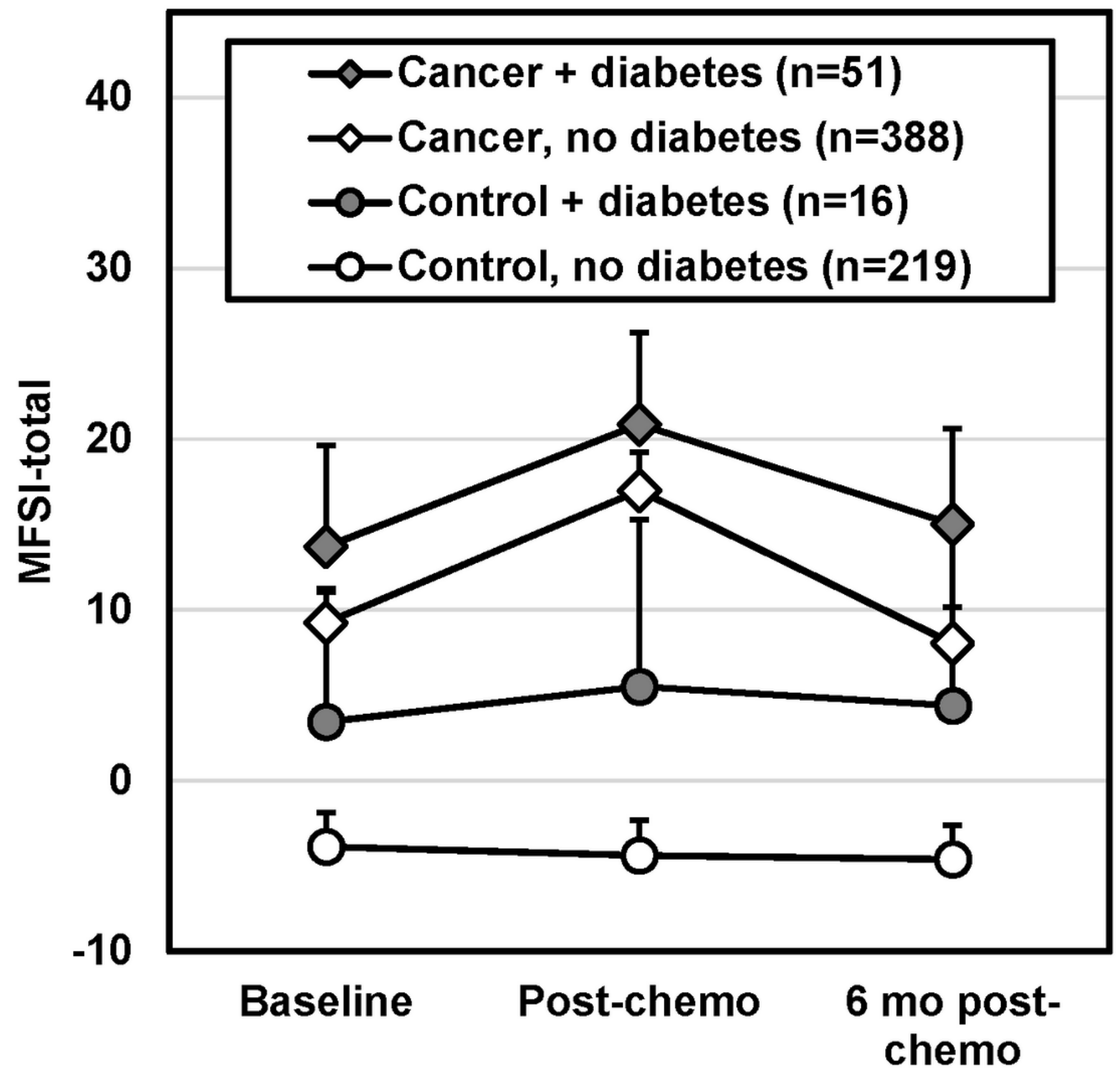

Figure 1 
Unadjusted total score for the Multidimensional Fatigue Symptom Inventory (MFSI) for patients with cancer (diamonds) and control participants without cancer (circles) from baseline (pre-chemotherapy) to postchemotherapy and 6 months post-chemotherapy, or equivalent time points for controls. Darkened markers indicate those with a diagnosis of diabetes and open markers are those without diabetes. There is a possible MFSI total score of -24 to 96 with a higher score indicating greater fatigue. Error bars depict $95 \%$ confidence interval. Figure was produced in Microsoft Excel 2016. 